\title{
Weak nonlinearities in viscoelastic mechanical properties of polymers near their glass transition: local vs. macroscopic laws for stress-induced acceleration of the mechanical response
}

\author{
Aude Belguise $^{1,2}$, Sabine Cantournet ${ }^{2}$, François Lequeux ${ }^{1}$, Hélène Montes ${ }^{1}$ \\ 1 UMR 7615, SIMM, ESPCI PSLCNRS UPMC, 10 Rue Vauquelin 75005 Paris \\ 2 PSL University, Mines ParisTech, Centre des Matériaux, CNRS 7633 BP 87, F-91003
}

\begin{abstract}
We focus on the role of dynamical heterogeneities on the weak mechanical nonlinearities of amorphous polymers near and above the glass transition temperature $\mathrm{T}_{\mathrm{g}}$ by combining experiments and numerical coarse-grained simulations. The acceleration of the macroscopic nonlinear modulus relaxation resulting from the applied stress is measured below yielding. As a result of dynamic disorder, the macroscopic acceleration differs from the local acceleration. We obtain a good agreement of experimental measurements with simulations computed by using an exponential function of the square stress for the local acceleration. Further, the length scale of dynamical heterogeneities is deduced.
\end{abstract}

Introduction

Many studies have focused on upscaling the mechanical response of disordered materials as it is encountered in many different systems (granular media [1], foams [2], networks [3], and polymers [4,5]) and situations (elasticity fracture [6] and yield stress [7]). Many nonintuitive behaviours have been reported, particularly in the nonlinear regime. However, change of scale in the nonlinear viscoelasticity of amorphous polymers has not yet been completely understood, particularly near the glass transition, where the dynamical heterogeneities are completely at stake.

Glassy polymers can be represented as tiled by domains of few tens of monomers whose collective configuration can be reorganised under thermal agitation [8-10]. Each domain has an intrinsic relaxation time that is temperature-dependent. Experiments have shown that the intrinsic relaxation times are randomly distributed over an entire system with a very wide distribution function (more than four decades) [9]. In this frame, numerical simulations 
showed that mechanical coupling between domains modifies the intrinsic local response of heterogeneities resulting in a complex macroscopic average of the local viscoelastic responses [11-15].

For a polymer glass near its glass transition temperature $\mathrm{Tg}$, relaxation times vary with temperature in the linear regime and with macroscopic stress in the nonlinear regime. In the linear regime, an increase in temperature results in a decrease in the local intrinsic times: the conformational changes are favoured by the thermal energy $\mathrm{k}_{\mathrm{B}} \mathrm{T}$. At the macroscopic scale, the time-dependent mechanical response of glassy polymers is accelerated. For instance, after a step strain, the macroscopic stress relaxes from its glassy value to its rubber value at shorter times for increasing temperature. However, the form of the macroscopic response does not significantly vary with temperature, i.e., in a good approximation, all intrinsic relaxation times are shifted by the same factor as temperature increases. This feature is known as the timetemperature superposition law. Thus, in the linear regime, the macroscopic stress relaxation measured at two different temperatures $\mathrm{T}$ and $\mathrm{T}_{\text {ref }}$ can be superimposed by applying a shift factor to the time scale which is equal to the factor applied to the local relaxation times. Thus, after an increase in temperature, the macroscopic and local mechanical responses are accelerated similarly.

Experiments have shown that the application of a nonlinear mechanical solicitation (strain or stress) results in an acceleration of the macroscopic response. For instance, after a step strain, the nonlinear stress relaxation of a glassy polymer is faster than that measured in the linear regime [16-22]. Therefore, nonlinear acceleration might be addressed with certain shift factors, as this approach is generally employed for the temperature effect. However, O'Connell et al. [21-22] have shown that nonlinear effects do not correspond to a simple shift factor applied to the time scale, as is the case for time-temperature superposition. Hence, the nonlinear acceleration of relaxation is challenging.

At the nanometric scale, plastic deformation is attributed to stress-induced molecular rearrangements [23-26] that occur by crossing energy barriers. Employing photobleaching methods, Lee et al. [27] measured the rotational correlation time as a function of the true stress for N,N0-dipentyl-3,4,9,10-perylenedicarboximide (DPPC) molecules in Polymethylmethacrylate (PMMA) samples near Tg during uniaxial creep deformation. According to Long et al. [28], these effects can be described by multiplying the intrinsic local relaxation times with a function $\mathrm{f}$ of the local stress $\sigma$ which is $\exp \left[-(\sigma / \mathrm{Y})^{2}\right]$, where $\mathrm{Y}$ is the 
critical stress given by $Y^{2}=\frac{2 k_{B} T G_{G}}{\xi^{3}}$, where $\xi$ is the size of the dynamical heterogeneities, $\mathrm{k}_{\mathrm{B}}$ is Boltzmann constant, $\mathrm{T}$ is the temperature, and $\mathrm{G}_{\mathrm{G}}$ is the glassy shear modulus.

In this study, we measure the macroscopic stress relaxation of cross-linked PMMA samples in the linear and weak nonlinear regimes. The experimental responses are analysed using a finite element approach that was developed in a previous study which mimics dynamical heterogeneities. In the nonlinear regime, intrinsic relaxation times characterising each heterogeneity are assumed to be proportional to the same function of the local stress according to the law proposed by Long et al. [28] To compare the local nonlinear response with the macroscopic response, we define a macroscopic acceleration function $\mathrm{F}$ which, is equal to the local function $\mathrm{f}$ if all domains have the same intrinsic relaxation time. Numerical simulations show that nonlinear responses of heterogeneous systems are different at the local and macroscopic scales, revealing the strong effect of disorder. Using Long's law as a local acceleration function, the shape of the experimental macroscopic acceleration function is in good agreement with that predicted by simulations. Finally, by comparing experimental and simulation results, we estimate the value of the critical stress $\mathrm{Y}$ involved in the local stress acceleration function of our PMMA sample, i.e., the only parameter we adjust to describe the macroscopic nonlinear response measured on PMMA samples. Thus, we estimate the size of dynamical heterogeneities using Long's model.

1. Materials and methods

We measured the macroscopic relaxation modulus of cross-linked PMMA chains at various temperatures in the glass transition domain.

\subsection{PMMA samples preparation}

PMMA samples were prepared according to the method described by Casas et al. [29]. A 1 $\mathrm{mm}$ sheet polymer was obtained using radical polymerisation and reticulation of methylmethacrylate monomers by applying UV irradiation for $8 \mathrm{~h}$. We chose diacrylate butanediol as the cross-linker. To initialise the polymerisation and reticulation, we added a photoinitiator (Irgacure (Ciba, France)) (0.1 wt\% monomer). The concentration of the crosslinker was chosen equal to $0.6 \%$ per mol of methylmethacrylate monomers such that the average weight between the cross-links Mc was similar to the entanglement distance Me. (Me $\approx 8000 \mathrm{~g} / \mathrm{mol}$, corresponding to an entanglement length of approximately $6 \mathrm{~nm}$ ). Cross- 
linking allowed us to successively perform step-strain linear and nonlinear tensile relaxation tests on the same sample.

\subsection{Procedure for stress relaxation measurements}

It is very difficult to precisely measure weak deviations to linearity below the yield point. Hence, the measurements of the linear and nonlinear responses should be conducted on the same sample in the same setup without displacing the sample. In practice, the macroscopic stress relaxations of PMMA samples are measured in the linear and weak nonlinear regimes at different temperatures. We verified that for strain amplitudes below $0.3 \%$, the mechanical responses are linear near Tg. Thus, we apply a step strain of $0.3 \%$ for linear measurements. The nonlinear stress relaxations are measured in the weak nonlinear regime, i.e., below the yield point. Thus, we applied a strain with an amplitude smaller than $2 \%$. To accurately observe the deviation of the nonlinear response with respect to the linear response, we applied a nonlinear condition strain ranging between $0.75 \%$ and $2 \%$.

Linear and nonlinear stress relaxations were successively measured on the same sample to compare them with sufficient accuracy. Between each measurement, thermal annealing was achieved by heating the sample at $32{ }^{\circ} \mathrm{C}$ above the glass transition temperature. The sample was then cooled to the temperature of the experiment while applying zero stress control. After attaining thermal equilibrium, the relaxation of the modulus was measured. We verified that the results obtained do not vary when an ageing step is added before the measurement.

2. Modelling the mechanical response of a polymer near its glass transition temperature

Amorphous polymers near their glass transition temperature are heterogeneous at the nanometric scale. To model nonlinear effects at the macroscopic scale on such a heterogeneous system, we use the 3D version of the model developed by Masurel et al. [3032]. The space is tiled by mechanically coupled domains. The resolution is performed in 3D using the finite element method [30].

The mechanical response of each domain is given by a Zener system made of two parallel branches. One branch consists of a Maxwell branch that represents the glassy contribution to the stress with an elastic modulus $\mathrm{E}_{\mathrm{G}}$ and a relaxation time of $\tau_{\mathrm{i}}$. The second branch has an elastic spring of modulus $E_{R}$ that represents the stress contribution due to the chain entropy. 
The glassy and rubber moduli are assumed to be equal for all domains. The compression modulus $\mathrm{K}$ is assumed to be independent of time and is set to $2 \mathrm{GPa}$ for the domains. We verified that for values of $\mathrm{K}$ larger than $1 \mathrm{GPa}$, the results of this study are same. Each domain has its own relaxation time $\tau_{\mathrm{i}}$ which is randomly drawn following a log-normal probability distribution, $P\left(\ln \left(\tau_{i}\right)\right)=\frac{1}{\sqrt{2 \pi s}} \exp \left(-\ln \left(\frac{\tau_{i}}{\tau_{0}}\right)^{2} / 2 s^{2}\right)$, where $\tau_{0}$ is the centre of the $\log$ normal distribution and $s$ is its width. $\tau_{0}$ is the only time scale in the model. The value of $\tau_{0}$ thus defines the position of the glass transition, whereas the width $s$ of the distribution controls the width of the glass transition.

In this study, nonlinear effects are modelled in the framework of the theory proposed by Long et al. For nonlinear simulations, the intrinsic relaxation time of domains is multiplied by the stress acceleration function predicted by Long et al. at the scale of heterogeneity and is equal to $f(\sigma)=\exp \left[-\left(\frac{\sigma}{Y}\right)^{2}\right]$. In the Long et al. theory, the stress is scalar. In this study, we extended the relation proposed by Long et al. to 3D tensor stress. According to the expression of $f(\sigma)$, intrinsic times are significantly accelerated for $\sigma>\mathrm{Y}$. The 3D criteria for polymer yielding follows an extended von Mises criterion [33]. Hence, we use the equivalent stress $\sigma^{e q}$ as:

$$
\sigma_{l o c}^{e q}=\sqrt{\frac{3}{2} \overline{\bar{\sigma}}^{D}: \overline{\bar{\sigma}}^{D}}-\alpha p
$$

with $\overline{\bar{\sigma}}^{D}$ the local deviatoric part of the stress and $\mathrm{p}$ its local pressure [30, 31]. The coefficient $\alpha$ is set to 0.3 in agreement with experiments [34].

In this study, numerical simulations were performed in 3D with systems containing $16 \times 16 \times$ 16 cubic domains which are mechanically coupled according to the finite element method. The resolution is performed using the finite element method with the finite element code Zebulon $[35,36]$. Each domain was divided into eight quadratic c3d20 cubic elements. To avoid edge effects, periodic boundary conditions were applied. The local stress used in the acceleration function in the nonlinear case is the mean over the domain. The macroscopic stress and strain are the means over the entire system.

We computed the relaxation of the system undergoing uniaxial elongation and applying either linear or nonlinear conditions. 


\section{Experimental results}

The modulus relaxation is measured in the linear regime at different temperatures by applying a step strain. The linear relaxation function of the modulus, called $\mathrm{E}_{\mathrm{L}}$, is determined by applying the time-temperature superposition principle. The data measured at temperature $\mathrm{T}$ are superimposed with the data measured at the reference temperature $T_{\text {ref }}$ by multiplying the time scale by a factor $a_{T / T r e f}$. Figure 1 presents the master curve we obtained which corresponds to the linear relaxation function $\mathrm{E}_{\mathrm{L}}$. Here, the reference temperature was chosen to be $108^{\circ} \mathrm{C}$.

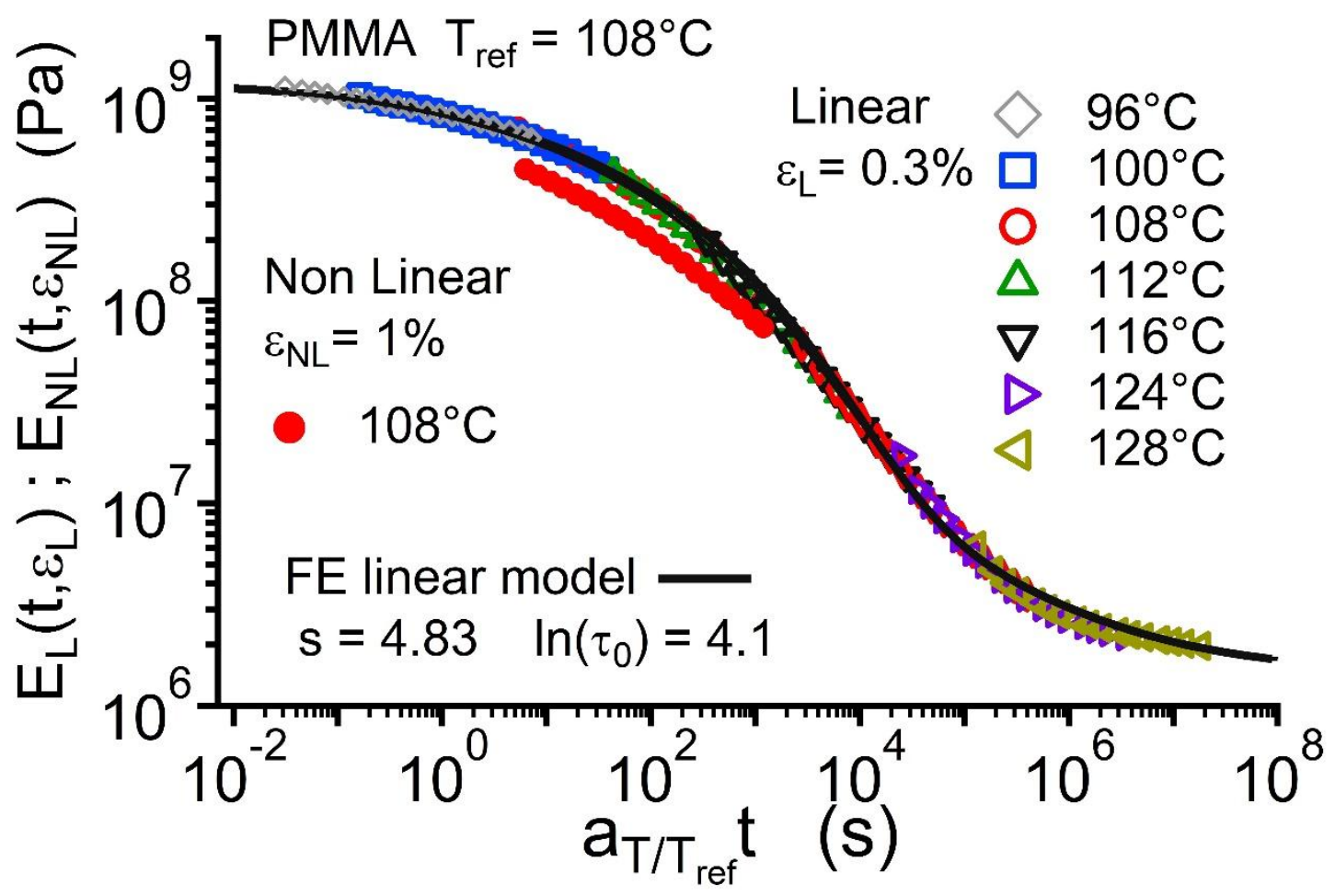

Figure 1: Linear relaxation master curve (empty markers) and nonlinear relaxation measured at 108 ${ }^{\circ} \mathrm{C}$ (filled circles). The black line corresponds to simulation predicted by our model for linear condition by applying the following values: $K=2 \mathrm{GPa}, E_{R}=1.5 \mathrm{MPa}, E_{G}=1.200 \mathrm{GPa}$, and $\ln \left(\tau_{0}\right)=$ $4.1 s=4.83$

On the same sample, we measured with the same setup the nonlinear response at different temperatures for a given strain amplitude. In figure 1, the nonlinear modulus $\mathrm{E}_{\mathrm{NL}}$, measured by applying a step strain of $1 \%$ at the reference temperature $\mathrm{T}_{\text {ref }}=108{ }^{\circ} \mathrm{C}$, is compared with 
the linear relaxation function $\mathrm{E}_{\mathrm{L}}$ at the same temperature. At $1 \%$ deformation, the macroscopic modulus relaxes faster than the modulus measured under linear conditions for the same sample.

Figure 2 presents the modulus relaxation measured in the nonlinear regime at different temperatures as a function of the variable $a_{T / T_{r e f}} t$, where $a_{T / T_{r e f}}$ are the time-temperature shift factors determined in the linear regime. As shown in figure 2, data measured in the nonlinear regime at different temperatures do not collapse into a master curve.

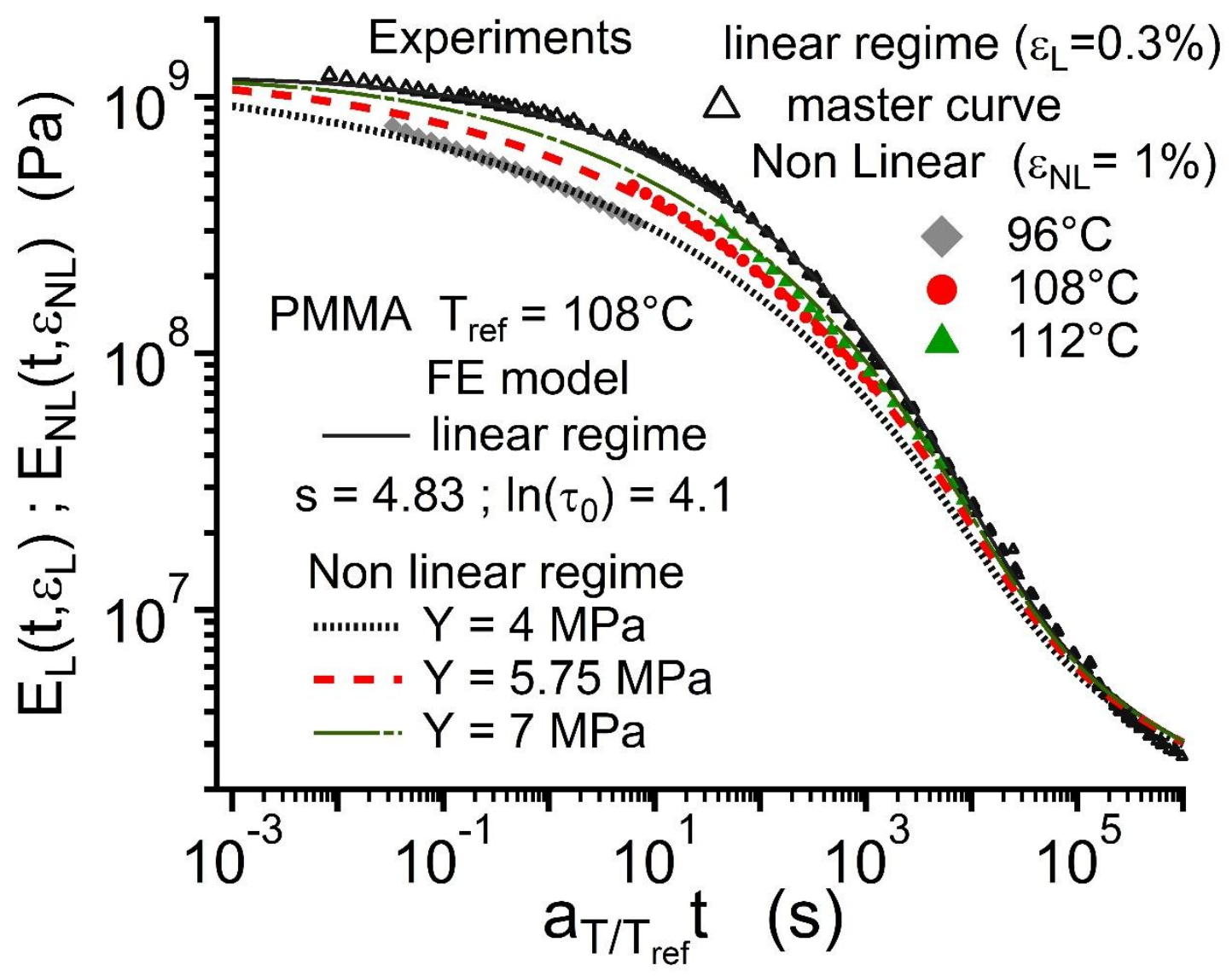

Figure 2: Nonlinear relaxation measured at different temperatures by applying a deformation of 1\% are plotted as a function of the shifted time $a_{T \pi r e f} t$ where $a_{T / T r e f}$ are the time-temperature equivalence shift factors measured in the linear regime at the reference temperature of $108^{\circ} \mathrm{C}$. The master curve measured in the linear regime is added. Experimental data are compared to numerical simulation predicted by our model in the linear and nonlinear regimes by applying: $E_{R}=1.5 \mathrm{MPa}, E_{G}=1.200$ $G P a, s=4.83, \ln \left(\tau_{0}\right)=4.1$. In the nonlinear regime, a good description of experiments is obtained adjusting the value of the critical stress $Y$ involved in the local acceleration function $f$ (see equation 2 ). For $\mathrm{T}=96{ }^{\circ} \mathrm{C}, \mathrm{Y}=4 \mathrm{MPa}$, for $\mathrm{T}=108^{\circ} \mathrm{C}, \mathrm{Y}=5.75 \mathrm{MPa}$, and for $\mathrm{T}=112^{\circ} \mathrm{C}, \mathrm{Y}=7 \mathrm{MPa}$. 
Consequently, we analyse the nonlinear response with respect to the linear response by comparing the nonlinear modulus relaxation to the master curve built in the linear regime at the same temperature. A comparison is thus performed for each temperature. The time scale accessible through the direct measurement of stress relaxation is significantly shorter than that related to the linear relaxation function $E_{L}$ which shows the complete relaxation of the modulus from its glassy value to its rubber value. Thus, the comparison of nonlinear and linear experimental data can be performed only on a limited (restricted) time range, and thus, a limited range of modulus values for each measurement temperature.

\section{Experiments via numerical approach}

We employ our numerical model to describe the linear and nonlinear responses measured at different temperatures on our PMMA samples.

\subsection{Linear regime}

First, we consider the experimental master curve $\mathrm{E}_{\mathrm{L}}$ built in the linear regime. The values of the glassy and rubber moduli, $\mathrm{E}_{\mathrm{G}}$ and $\mathrm{E}_{\mathrm{R}}$, of each heterogeneity are equal to the macroscopic moduli measured on our samples, i.e., $\mathrm{E}_{\mathrm{G}}=1.2 \mathrm{GPa}$ and $\mathrm{E}_{\mathrm{R}}=1.5 \mathrm{MPa}$. Further, for these

simulations, $\mathrm{K}=2 \mathrm{GPa}$. Consequently, the value of the Poisson coefficient $v$ is equal to 0.4999 in the rubber state and to 0.40 in the glassy state; these values are in agreement with the experimental values of $v$ reported for PMMA, that are ranged between 0.34 and 0.4 [37]. The fitting of the master curve using the numerical model is performed by adjusting the values of the width $s$ and centre $\tau_{0}$ of the intrinsic relaxation time distribution. At the reference temperature of $108{ }^{\circ} \mathrm{C}$, we obtained a good agreement between the simulations and experiment when $\mathrm{s}=4,83$ and $\tau_{0}=60 \mathrm{~s}$ with $\mathrm{E}_{\mathrm{G}}=1.2 \mathrm{GPa}, \mathrm{E}_{\mathrm{R}}=1.5 \mathrm{MPa}$, and $\mathrm{K}=2 \mathrm{GPa}$.

By employing the values of the five parameters determined from data measured in the linear regime, we now model the nonlinear response measured on the same PMMA samples.

\subsection{Nonlinear regime}

We now compare the predictions of our model by applying nonlinear conditions to experimental results measured on the PMMA samples. We recall that according to our numerical approach, the intrinsic relaxation time of each heterogeneity is multiplied in the 
nonlinear regime by a local stress acceleration function that we assume to be equal to the function that was theoretically predicted by Long et al., i.e.:

$$
f\left(\sigma_{l o c}^{e q}\right)=\exp \left[-\left(\frac{\sigma_{l o c}^{e q}}{Y}\right)^{2}\right]
$$

where the local equivalent stress $\sigma_{l o c}^{e q}$ is given by equation 1 .

Computations were performed using the values of $\mathrm{E}_{\mathrm{R}}, \mathrm{E}_{\mathrm{G}}, \mathrm{s}$, and $\tau_{0}$ obtained from the fitting of the linear response measured on our PMMA samples. The critical stress $\mathrm{Y}$ is thus a parameter that must be adjusted.

Using $\mathrm{Y}=5.75 \mathrm{MPa}$, we obtained a good agreement between the simulations and nonlinear response measured at the reference temperature of $108{ }^{\circ} \mathrm{C}$ and for a strain step of $1 \%$ amplitude. The simulation and experimental data are compared in figure 2.

If $\mathrm{Y}$ is assumed to be independent of temperature, the model predicts a time-temperature superposition for the nonlinear response for a given strain amplitude. Because no timetemperature superposition in the nonlinear regime is experimentally observed, the value of $\mathrm{Y}$ must be varied with temperature.

Figure 2 compares the experiments performed for $1 \%$ strain at different temperatures to the numerical curves predicted by our model in the nonlinear regime assuming a temperature dependence of $\mathrm{Y}$. We adjust the value of $\mathrm{Y}$ to obtain the best description of the experimental result. We found values for $\mathrm{Y}$ varying from 4 to $7 \mathrm{MPa}$ for temperatures ranging between 96 ${ }^{\circ} \mathrm{C}$ and $112{ }^{\circ} \mathrm{C}$, as shown in figure 3 . 


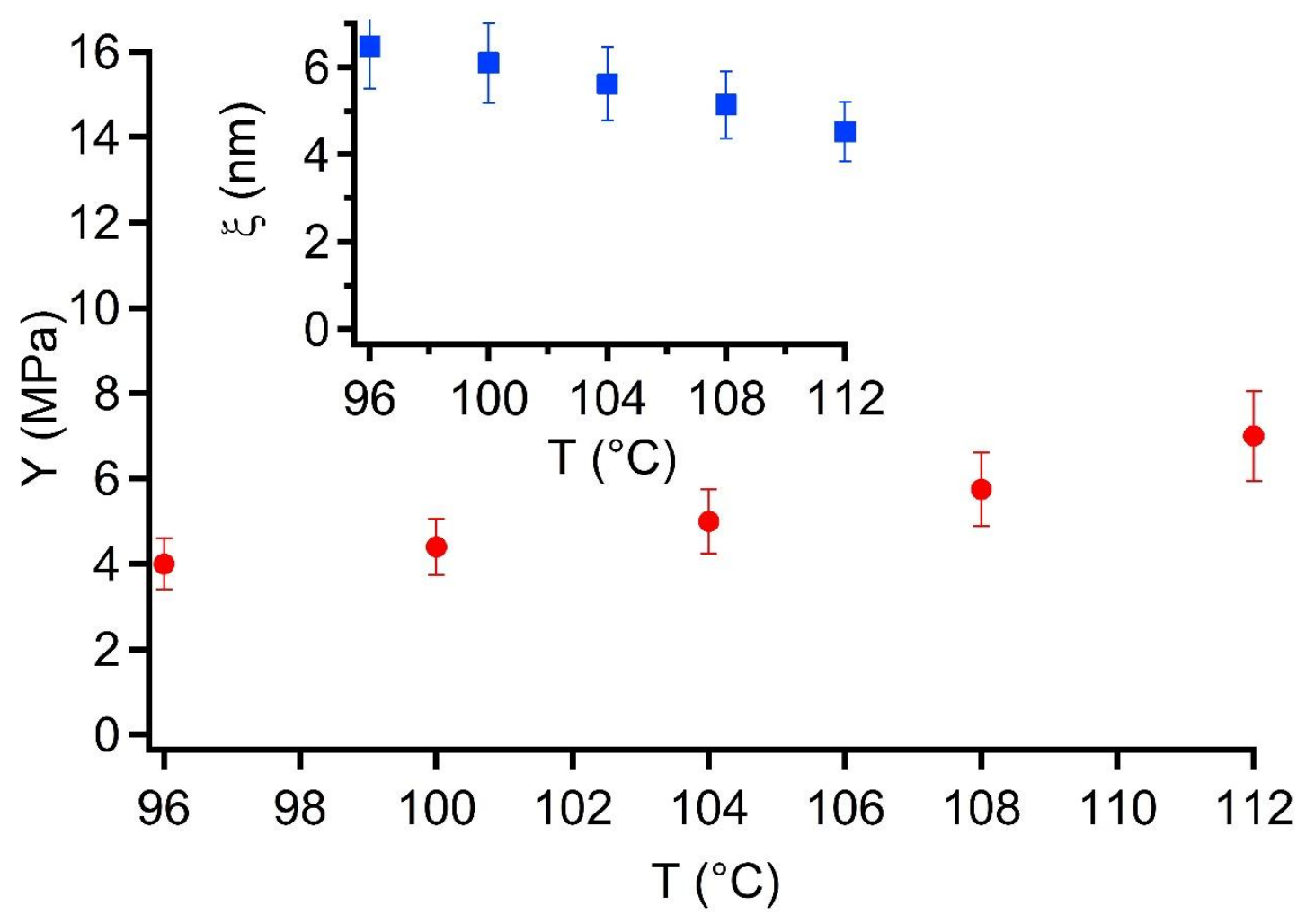

Figure 3: Parameter $Y$ as a function of temperature inferred from the fitting by our model of the nonlinear responses measured at different temperatures applying $1 \%$ step strain on PMMA samples (see figure 2). The corresponding values of the size of heterogeneities computed following the relation suggested by Long et al. [28] are presented in the inset.

We analysed the distribution of local strain during nonlinear relaxation. In such heterogeneous systems, the strain is locally distributed with domains undergoing a larger local strain than the other. However, we observed that the local strain is always smaller than $4 \%$ for a $1 \%$ macroscopic strain applied. In the strain range studied in this study, the local stress is not modified by the limit extensibility of polymer chains; an extension of $4 \%$ at the length scale of heterogeneity (i.e., a few nanometres) is significantly smaller than the maximal extension at this scale, which is given by $\varepsilon_{\max }=1-\frac{R_{\max }}{\left\langle R_{e e}^{2}\right\rangle^{\frac{1}{2}}}=1-\frac{b N}{b \sqrt{N}}=1-$ $\sqrt{N}$, where $\mathrm{R}_{\max }=\mathrm{bN}$ is the contour length of a polymer chain, $\left\langle R_{e e}^{2}\right\rangle=b \sqrt{N}$ is its meansquare end-to-end distance, $\mathrm{b}$ is the Kühn length, and $\mathrm{N}$ is the number of Kühn segments of a chain segment included in heterogeneity [38 ]. For a size of heterogeneity of $3 \mathrm{~nm}$, applying b $=1.7 \mathrm{~nm}$ for PMMA chains, the approximate value of $\mathrm{N}=(3 / 1.7)^{2}=3.5$, leading to a maximum value of approximately $\varepsilon_{\max }=76 \%$ for PMMA chains. The extension undergone 
by the chain segment is thus negligible at the scale of heterogeneities. The local response we assume in our model is thus valid in the weak nonlinear regime.

Our model provides a satisfactory description of macroscopic response in the linear and weak nonlinear regimes. The model provides also information on the local stress field and the local relaxation times distribution during the nonlinear macroscopic stress relaxation.

We analyse the distribution of local relaxation times that are defined as $\tau_{i}\left(\sigma_{l o c}^{e q}\right)=\tau_{i} f\left(\sigma_{l o c}^{e q}\right)$ during the stress relaxation for nonlinear conditions. Figure 4 presents the distribution functions of the napierian logarithm of the relaxation times $P\left(\ln \left(\tau_{i}\left(\sigma_{l o c}^{e q}\right)\right)\right)$ at different steps of the nonlinear relaxation, computed with $\mathrm{Y}=6 \mathrm{MPa}$ and $\varepsilon=0.01$. The corresponding macroscopic stress relaxation is shown in figure 5-a. We can identify several regimes.

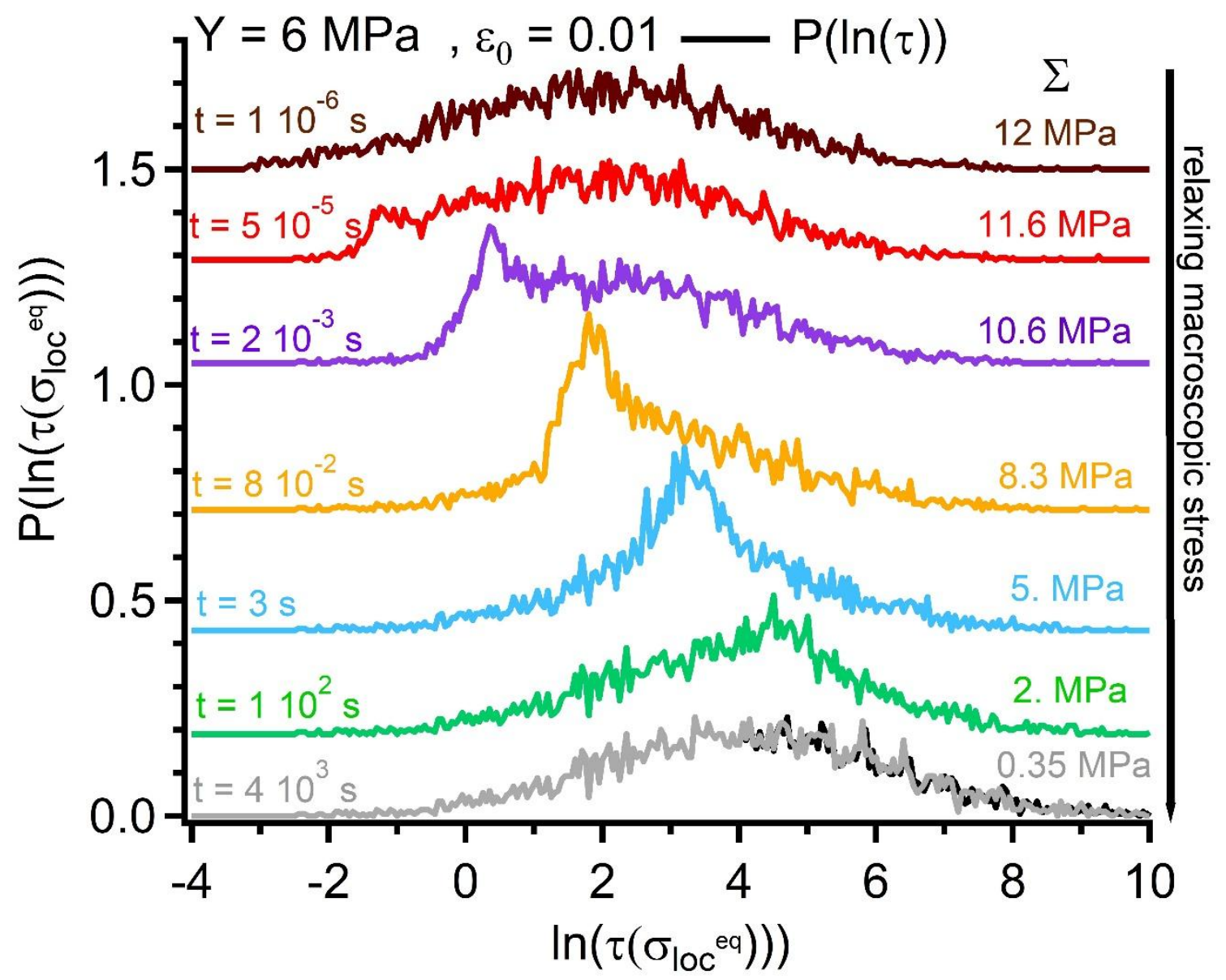

Figure 4: distribution function of the napierian logarithm of local relaxation times $\ln \left(\tau_{i}\left(\sigma_{\text {loc }}^{\text {eq }}\right)\right)$ at different steps of the macroscopic stress relaxation. Computation were performed applying $Y=6 \mathrm{MPa}, \varepsilon_{0}=0.01$. The width and the center time of the intrinsic 
relaxation time distribution were equal to $s=4.23$ and $\tau_{0}=60 \mathrm{~s}$. The value of the macroscopic stress relaxation $\Sigma$ are reported for each time. The corresponding whole macroscopic stress relaxation curve $\Sigma$ is presented in figure 5-a. Each curve is arbitrarily shifted by a constant.

At short macroscopic times, $\left(\mathrm{t}<1 \mathrm{e}^{-6} \mathrm{~s}\right.$ in figures 4 and $\left.5-\mathrm{a}\right)$, the shape of the distribution function does not change compared to the shape of the intrinsic relaxation time distribution, but the distribution is shifted towards lower times. The shift results from the homogenous stress just after application of step strain when all the domains are in their glassy state.

In a second stage and at the beginning of the nonlinear stress relaxation $\left(\mathrm{t}=5 \mathrm{e}^{-5} \mathrm{~s}\right.$ and $\mathrm{t}=2 \mathrm{e}^{-3} \mathrm{~s}$ in figures 4 and 5-a), the domains having the shortest intrinsic relaxation times relax their local stresses. Consequently, $\sigma_{l o c}^{e q}$ relaxes towards zero, and their relaxation times $\tau_{i}\left(\sigma_{l o c}^{e q}\right)$ increase up to the intrinsic relaxation time of the domain. In the same time, the slowest domains still undergo a large local stress. The values of their local relaxation times stay shifted towards low values. As a result, the fastest domains are shifted towards larger times, while the slowest ones remain unchanged and sustain most of the stress [12]. A growing peak appears on the fast side of the distribution function that corresponds to the accumulation of the relaxation times of fast relaxing and relaxed domains. Consequently, the width of the distribution decreases.

For macroscopic stress value of the order of $E_{0} \varepsilon /$ e (i.e. $t=3 \mathrm{~s}$ and $\Sigma=5 \mathrm{MPa}$ in figures 4 and 5-a), the network constituted by the slowest domains vanishes : the local stresses of the latter domains relax and their local relaxation times $\tau_{i}\left(\sigma_{l o c}^{e q}\right)$ tend towards their values at rest. As a result, the width of the distribution function increases again up to its initial value. At long macroscopic times $\left(\mathrm{t}>4 \mathrm{e}^{3} \mathrm{~s}\right)$, all domains have relaxed their stress and the local relaxation time distribution converges towards the distribution of the intrinsic relaxation times.

The inverse of the width of the time distribution 1/s is plotted in Figure 5-a as function of time for varying values of $\mathrm{Y}$. The width $\mathrm{s}$ is determined applying the relation : $S^{2}=\frac{\int\left(\ln \left(\tau_{i}\left(\sigma_{l o c}^{e q}\right)\right)-\left\langle\ln \left(\tau_{i}\left(\sigma_{\text {loc }}^{e q}\right)\right)\right\rangle\right)^{2} P\left(\ln \left(\tau_{i}\left(\sigma_{\text {loc }}^{e q}\right)\right)\right) d \ln \left(\tau_{i}\left(\sigma_{\text {loc }}^{e q}\right)\right)}{\int P\left(\ln \left(\tau_{i}\left(\sigma_{\text {loc }}^{e q}\right)\right)\right) d \ln \left(\tau_{i}\left(\sigma_{\text {loc }}^{e q}\right)\right)}$. Figure 5-b presents the variation of the geometric mean value of the relaxation times modified by the local stress $\left\langle\tau_{i}\left(\sigma_{l o c}^{e q}\right)\right\rangle$ divided by the geometric mean of the intrinsic time distribution $\tau_{0}$ as a function of the macroscopic stress. The width of the local relaxation time distribution reaches a minimum 
value for a macroscopic stress value of the order of $\sigma_{0} / \mathrm{e}$ corresponding to the end of the percolation of slow domains.

The evolution of the local relaxation times predicted by our numerical model for nonlinear stress relaxation agrees with observations reported by Lee et al [19] for nonlinear creep experiments on PMMA samples: they not only observed a decrease of the local mean relaxation time under stress, previously discussed by Long et al [28], but also a narrowing of the local time distribution followed by its broadening after the flow onset.

Our numerical approach shows that mechanical percolation drives the nonlinear macroscopic stress relaxation in our PMMA samples. We emphasize the main difference between stepstrain and creep experiments is that, in the first case, the mechanical percolation threshold is crossed keeping a small macroscopic strain i.e. smaller than the yield strain. In contrast in creep experiments, the strain continuously increases. Having this effect in mind, we only performed and discussed step strain experiments, in order to remain in the small strain regime, and to avoid effects due to limit extensibility.
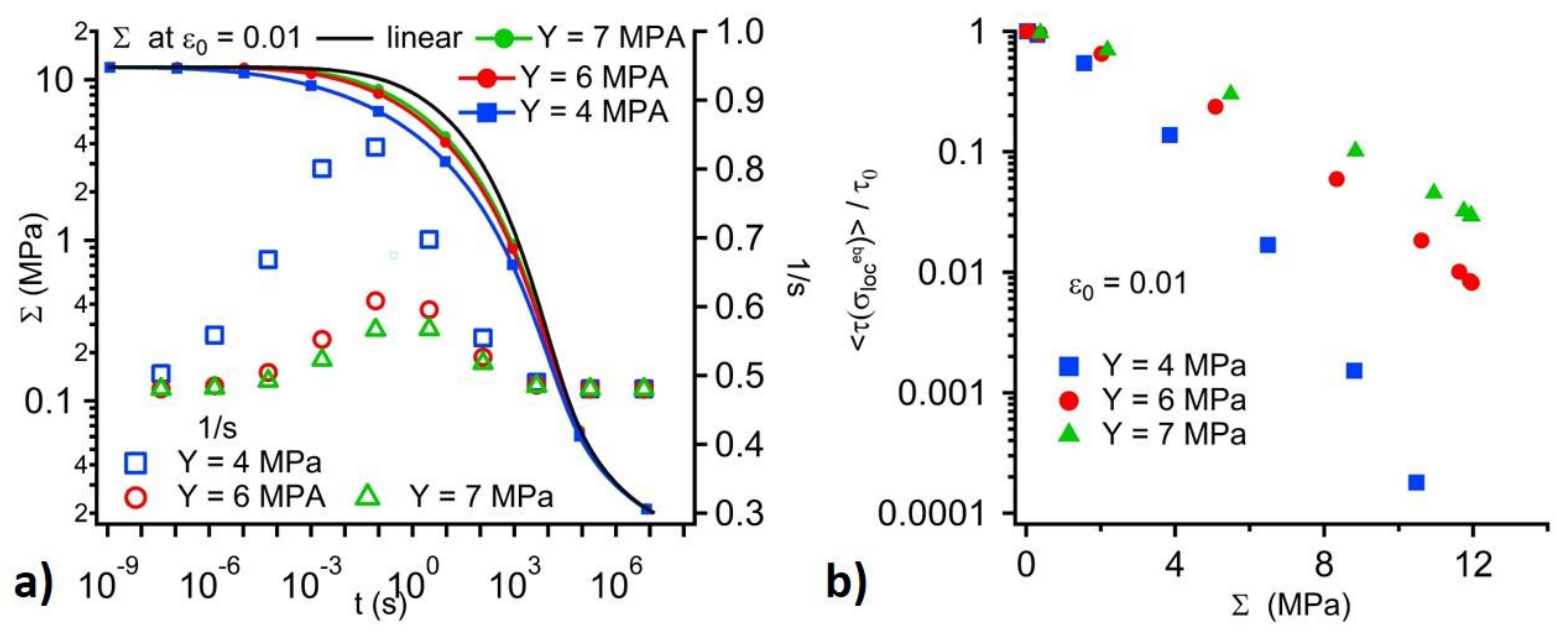

Figure 5: a) macroscopic stress relaxation $\Sigma$ as a function of macroscopic time. Computation were performed for $\varepsilon_{0}=0.01$ and for varying $Y$ values. The inverse of the width of the corresponding local relaxation time distributions $1 / \mathrm{s}$ is shown as a function of macroscopic time. $b$ ) the geometric mean time of the corresponding local relaxation time distributions $\left\langle\tau_{i}\left(\sigma_{\text {loc }}^{\text {eq }}\right)\right\rangle$ divided by the geometric mean time value of the intrinsic time distribution $\tau_{0}$ is plotted as a function of the macroscopic stress $\Sigma$ 
Now that we have shown that our model is able to describe the feature observed by Lee et al [19] at a microscopic scale, we turn to the comparison of behaviours of the macroscopic stress.

5. Comparison of macroscopic and local stress relaxations

As shown in figure 2, the nonlinear relaxation is accelerated when compared with the linear relaxation. To analyse the effect of disorder on the nonlinear response, we first define a macroscopic acceleration function $F$ which we will compare with the local function $f$ that is applied at the scale of heterogeneities.

\subsection{Definition of the macroscopic acceleration function}

We use the macroscopic linear and nonlinear response of a system to define a macroscopic acceleration function $\mathrm{F}$ such that, for a single Zener system, it is equal to the local stress acceleration function $\mathrm{f}$.

First, we consider a single Zener system with a Maxwell branch representing the glassy contribution to the stress with an elastic modulus $\mathrm{E}_{\mathrm{G}}{ }^{\mathrm{Z}}$ and relaxation time $\tau_{0}$ that is in parallel with an elastic spring of modulus $\mathrm{E}_{\mathrm{R}}{ }^{\mathrm{z}}$, which represents the stress contribution owing to the chain entropy. In our approach, we model the nonlinear behaviour of the polymer by multiplying the relaxation time $\tau_{0}$ with an acceleration function $f$ of the stress $\sigma$. According to Long et al., we assume an acceleration function of the form $\exp \left[-(\sigma / Y)^{2}\right]$.

For a step strain, the relaxation of the modulus in the linear regime is given in $1 \mathrm{D}$ by

$\frac{d E_{L}^{Z}}{d t}=-\frac{E_{L}^{Z}(t)-E_{R}^{Z}}{\tau_{0}}$

and in the nonlinear regime by:

$\frac{d E_{N L}^{Z}}{d t}=-\frac{E_{N L}^{Z}(t)-E_{R}^{Z}}{\tau_{0} f\left(E_{N L} \varepsilon_{0}\right)}$

In equations 3 and $4, E_{L}^{Z}$ and $E_{N L}^{Z}$ are the relaxation moduli (i.e., the ratio of stress over strain), for linear and nonlinear behaviours, respectively, and $\varepsilon_{0}$ is the deformation. Further, the equations driving the modulus relaxation are written in 1D. They can also be written in 3D as 
shown in Annex 1. The 1D and 3D relaxation equations result in the same equations regarding the direction of the deformation (uniaxial elongation in this study).

From equations 3 and 4, we deduce that

$\frac{\frac{d E_{L}^{Z}}{d t}}{\frac{d E_{N L}^{Z}}{d t}}=f\left(E, \varepsilon_{0}\right)$

where the two time derivatives of the L.H.S. of equation 3 are considered not at the same time but for the same values of $\mathrm{E}_{\mathrm{L}}{ }^{\mathrm{z}}$ and $\mathrm{E}_{\mathrm{NL}}{ }^{\mathrm{z}}$ that are equal to $\mathrm{E}$.

We extend equation 5 to the macroscopic system, and thus, define the macroscopic acceleration function $F$ as the ratio of the derivatives of linear $\left(\mathrm{E}_{\mathrm{L}}\right)$ and nonlinear relaxations $\left(\mathrm{E}_{\mathrm{NL}}\right)$ measured at the same state of relaxation:

$F\left(E, \varepsilon_{0}\right)=\frac{d E_{L}}{d t} / \frac{d E_{N L}}{d t}$

with $E_{L}=E_{N L}=E$. The function $F$ depends on the relaxation state which is characterised by the value of the modulus and strain that has been applied.

According to the definition we choose for F, for a single Zener system, the macroscopic function $\mathrm{F}$ depends only on the stress undergone by the Zener system that is equal to $\mathrm{E}_{\mathrm{NL}} \varepsilon_{0}$.

In the next section, we show that because of disorder, the macroscopic acceleration function $\mathrm{F}$ deviates from the local function $\mathrm{f}$. Further, we analyse the shape of the macroscopic acceleration function F resulting from measurements performed on our PMMA samples.

\subsection{Shape of the macroscopic acceleration function $F$}

\subsubsection{Experimental results}

Applying equation 5, the experimental macroscopic acceleration function of our PMMA samples is determined from the linear master curve and nonlinear response measured at the same temperature T. Figure 6 presents the macroscopic acceleration functions obtained at each temperature for a step strain of $1 \%$ amplitude. We observed that the macroscopic function of our samples, $\mathrm{F}$, depends on temperature. We represent the macroscopic function $\mathrm{F}$ by plotting $-\ln \left[\mathrm{F}\left(\mathrm{E}, \varepsilon_{0}\right)\right]$ as a function of the macroscopic stress $\mathrm{E} \varepsilon_{0}$ with log scales. Here, we 
obtained a linear curve with a slope of approximately $0.9+/-0.1$. The value of the slope does not significantly vary with temperature in the range probed by experiments.

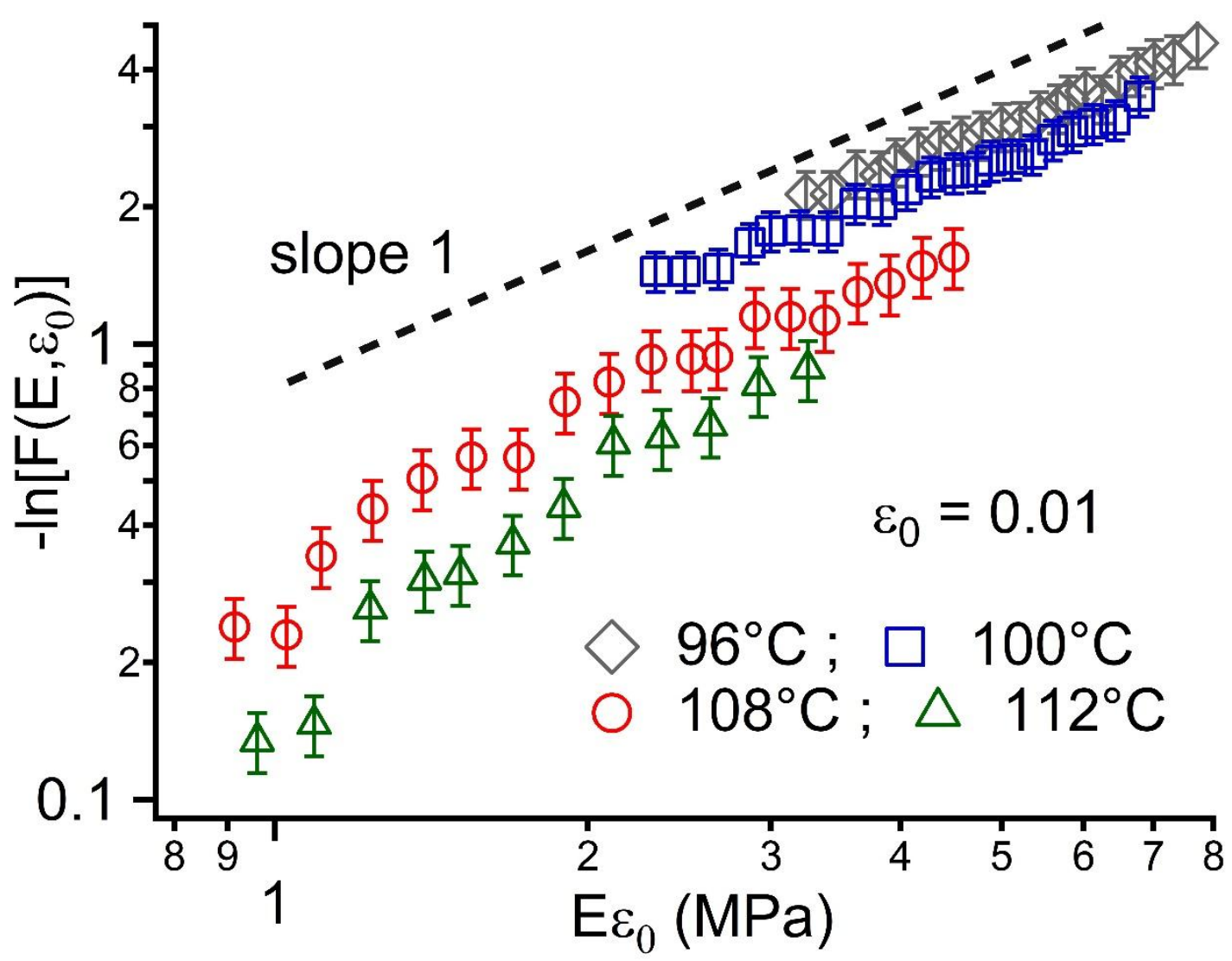

Figure 6: The quantity $-\ln \left[F\left(E, \varepsilon_{0}\right)\right]$ deduced from experiments performed at different temperatures applying a deformation $\varepsilon_{0}$ of $1 \%$ vs. macroscopic stress E $\varepsilon_{0}$ in log-log scale

Thus, on the modulus range accessible by experiments, the form of $F$ can be described by the relation $F\left(E, \varepsilon_{0}\right)=\exp \left[-(E / Z)^{\mathrm{m}}\right]$ with $\mathrm{Z}$ that depends on temperature. To understand the temperature and modulus dependence of $\mathrm{F}$ observed experimentally, we compare the experimental results with numerical responses predicted by our model.

\subsubsection{Numerical predictions}

We compute the macroscopic acceleration function $F$ for a disordered system and compare it with the acceleration function $f$ of a Zener system. Figure 7 presents the curves obtained by 
plotting $-\ln (F)$ and $-\ln (f)$ as a function of $\mathrm{E} / \mathrm{E}_{0}$ on a $\log$ scale for $\mathrm{Y}=5 \mathrm{MPa}$ with $\mathrm{E}_{0}=\mathrm{E}_{\mathrm{G}}+\mathrm{E}_{\mathrm{R}}$. Here, $\mathrm{F}$ and $\mathrm{f}$ exhibit very different behaviours.

First, we consider the case of a single Zener system. Here, $-\ln (f)=\left(E \varepsilon_{0} / Y\right)^{2}$ results in a straight line with a slope of 2 on a $\log$ scale.

In the heterogeneous case, we identify a range of modulus values over which the quantity $\ln \left(\mathrm{F}\left(\mathrm{E}, \varepsilon_{0}\right)\right)$ varies linearly with $\mathrm{E} / \mathrm{E}_{0}$ in log scale. In this modulus range, the slope is equal to 0.83. This means that owing to dynamical heterogeneities, the macroscopic and local acceleration functions are different.

However, the local and macroscopic acceleration would have been equal if the local acceleration function was assumed to depend on the mean stress rather than the local stress. The difference between the macroscopic and local acceleration functions occurs because the stress field is disordered. However, at short times, immediately after the strain step where $\mathrm{E} / \mathrm{E}_{0}$ is close to 1 , the stress field is homogeneous and $f$ and $F$ collapse. With increasing time, a disordered stress field occurs owing to the distribution of intrinsic times. This stress field results in heterogeneous acceleration. The macroscopic acceleration deviates from the local acceleration.

In practice, the range of modulus values over which the quantity $\ln \left(\mathrm{F}\left(\mathrm{E}, \varepsilon_{0}\right)\right)$ varies linearly with $\mathrm{E} / \mathrm{E}_{0}$ in $\log$ scale corresponds to the experimental windows for which mechanical measurements are sufficiently accurate to experimentally determine the acceleration function. Thus, we focus on this modulus range, where the form of the macroscopic acceleration function is given by $e^{-\left(\frac{E}{z}\right)^{m}}$. 


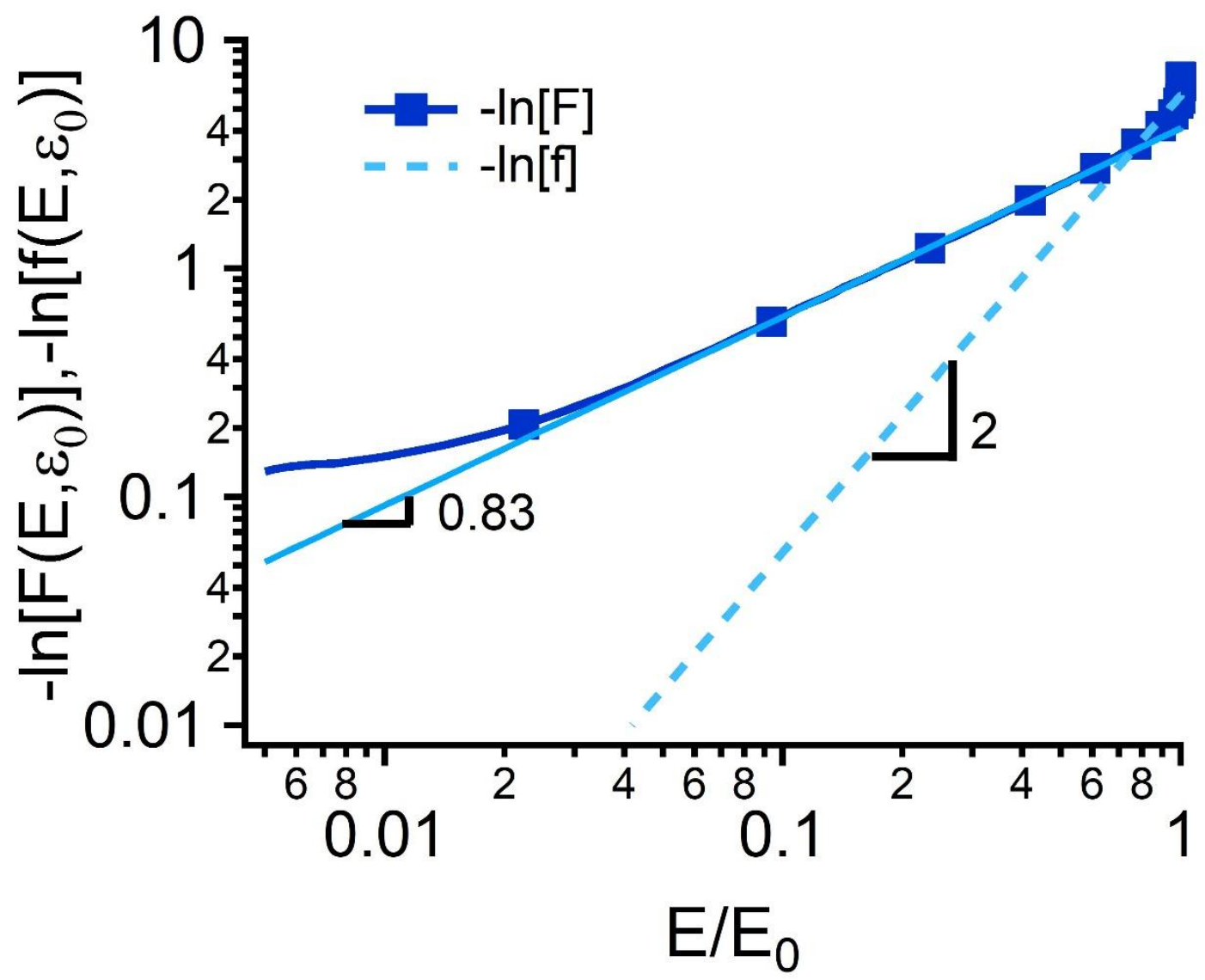

Figure 7: The quantity $-\ln \left[F\left(E, \varepsilon_{0}\right)\right]$ as a function of the modulus normalised by $E_{0}$, the modulus at $t=0$ in log-log scale. Data in filled blue squares are computed assuming a $3 D$ heterogeneous system applying a local acceleration function $f$ given by equation 2 with $Y=5 M P a$

\subsubsection{Dependence of $F$ on strain amplitude}

First, we compare the macroscopic acceleration functions resulting from simulations performed by applying different strain amplitudes $\varepsilon_{0}$. The curves obtained for different $\varepsilon_{0}$ values have similar shapes as shown in figure 8-a. The value of the slope of the linear part of the curve $\mathrm{m}$ is not significantly dependent on the strain amplitude over the deformation range probed in this study. As a result, the curves can be overlaid by applying a vertical shift factor $1 / \mathrm{g}\left(\varepsilon_{0}\right)$, as presented in figure 8 -b. Therefore, although the amplitude of the macroscopic acceleration function varies with strain amplitude, the value of the slope $\mathrm{m}$ does not depend on $\varepsilon_{0}$ at zero order. 


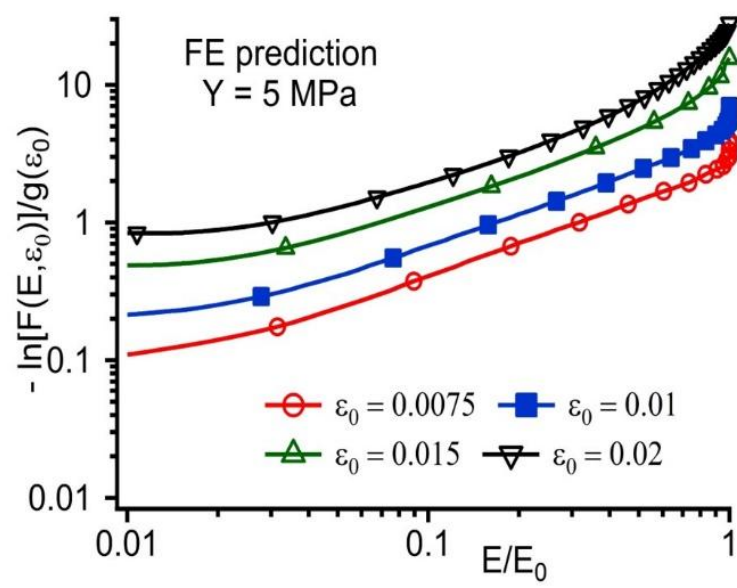

a)

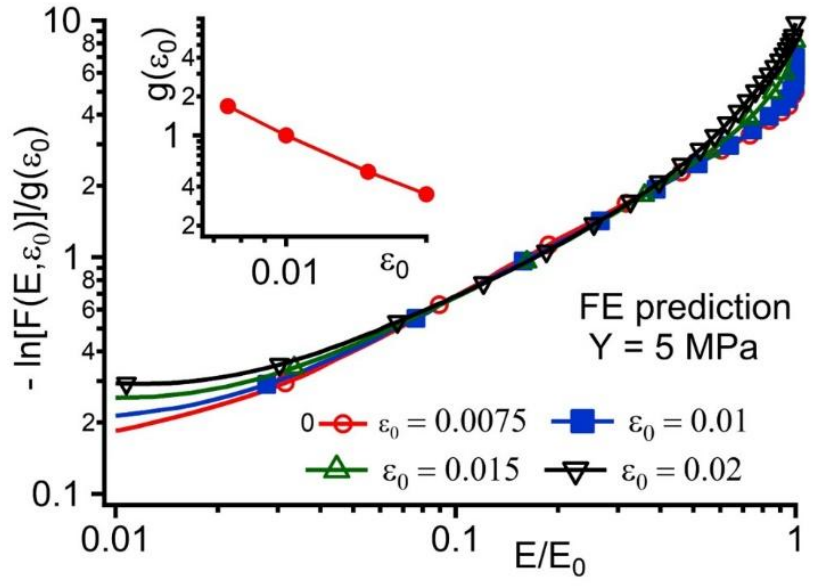

b)

Figure 8: (a) $-\ln \left(F\left(E, \varepsilon_{0}\right)\right)$ as a function of $E$ for different strains in log scales. (b) vertical shift factors $1 / g\left(\varepsilon_{0}\right)$ are applied to the data. The inset presents the variation of $g\left(\varepsilon_{0}\right)$ as a function of the strain amplitude in log scales

\subsubsection{Dependence on $Y$}

Variations in the parameter $\mathrm{Y}$ of the local acceleration function lead to vertical shifts of the curves, as illustrated in figure 9. The vertical shift factor b, which should be applied to overlay the curves in the modulus range we consider, is proportional to $\mathrm{Y}^{1.87 \pm 0.1}$, as shown in the inset of figure 9-b. According to the Long model, the parameter $\mathrm{Y}$ depends on the temperature and size of dynamical heterogeneities.

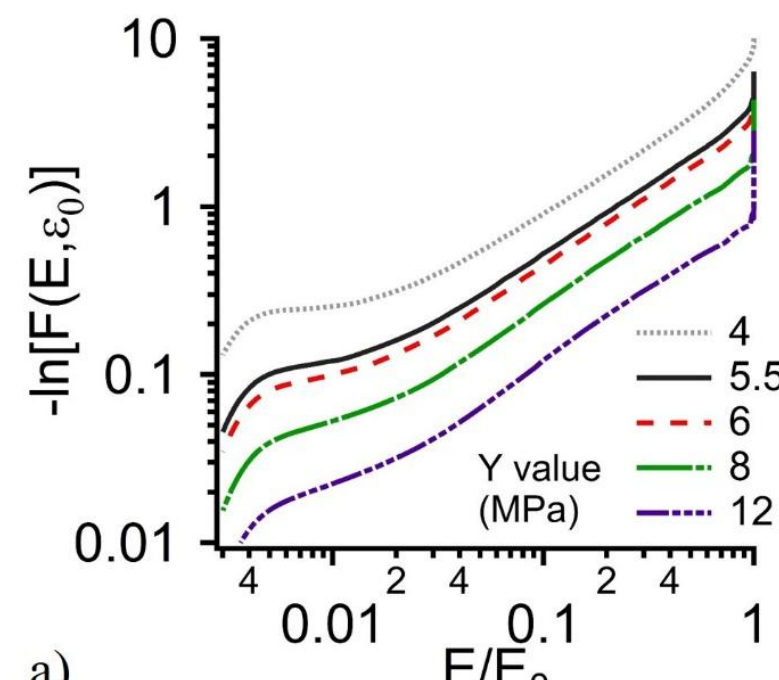

a) $\mathrm{E} / \mathrm{E}_{0}$

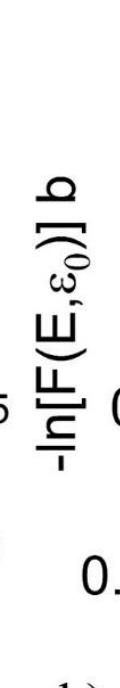

b)

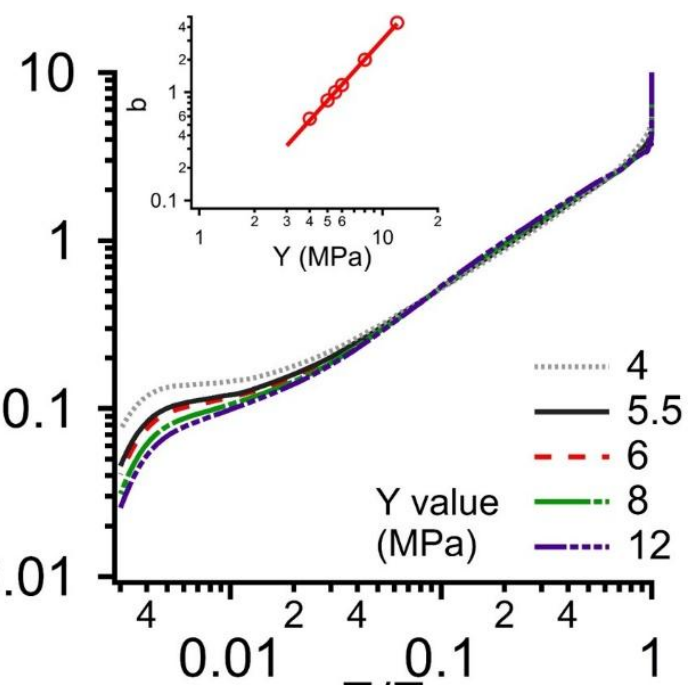


Figure 9: a) The quantity - $\ln \left(F\left(E, \varepsilon_{0}\right)\right)$ predicted by our model for varying values of the critical stress $Y$ are plotted as a function of the modulus value E divided by the modulus value in the glassy state $E_{0}$ in log-log scale. Computations were performed by applying: $E_{R}=1.5 \mathrm{MPa}$, $E_{G}=1.2 \mathrm{GPa}, s=4.83, \ln \left(\tau_{0}\right)=4.1$ and $\varepsilon=1 \%$. Y value was varied from $3 \mathrm{MPa}$ to $12 \mathrm{MPa}$. b) numerical curves collapse if a shift factor $b$ is applied to the quantity $-\ln \left(F\left(E, \varepsilon_{0}\right)\right)$. The inset presents the variation of $b$ as a function of $Y$ in $\log -\log$ scale. The line corresponds of $a$ power law function with an exponent equal to 1.87.

As shown in figure 2, we obtain a very good fitting of experimental data employing at the local scale the acceleration function predicted by Long et al. However, we studied the variation in the shape of the macroscopic acceleration function $\mathrm{F}$ of disordered systems for more general local acceleration functions of the form of $\exp \left[-(\sigma / Y)^{\mathrm{n}}\right]$. Computations were performed for values of exponent $n$ varying from 1 to 3 . The results are presented in detail in Annex 2. The effect of the width of the relaxation time distribution is also studied. Despite the value of $\mathrm{n}$ assumed for the local acceleration function, we observed that there is always a modulus range over which the quantity $-\ln \left[\mathrm{F}\left(\mathrm{E}, \varepsilon_{0}\right)\right]$ scales as $\left(E / E_{0}\right)^{\mathrm{m}}$, where $\mathrm{m}$ depends on $\mathrm{n}$. The experimental value of $m=0.9$ corresponds to the values of $n \gtrsim 2$.

5.3. Origin of the temperature dependence of the experimental macroscopic acceleration function

The experimental curves obtained by plotting $-\ln \left[\mathrm{F}\left(\mathrm{E}, \varepsilon_{0}\right)\right]$ as a function of $\mathrm{E}$ are linear and depend on the temperature of the experiment. The temperature dependence of $\mathrm{F}$ observed for our PMMA samples could result from the temperature dependence of the critical stress $Y$. The macroscopic acceleration functions measured at different temperatures should thus be overlaid by applying a vertical shift factor resulting from the temperature dependence of Y. Figure 10 presents the experimental master curves similarly obtained for $1 \%$ and $1.5 \%$ deformation. Similar slopes were observed for the two deformation amplitudes, as was the case in our simulations. As shown in figure 10, the macroscopic acceleration functions measured for a reference temperature of $108{ }^{\circ} \mathrm{C}$ and $1 \%$ and $1.5 \%$ strain can be described using $\mathrm{Y}=5.75 \mathrm{MPa}$. 


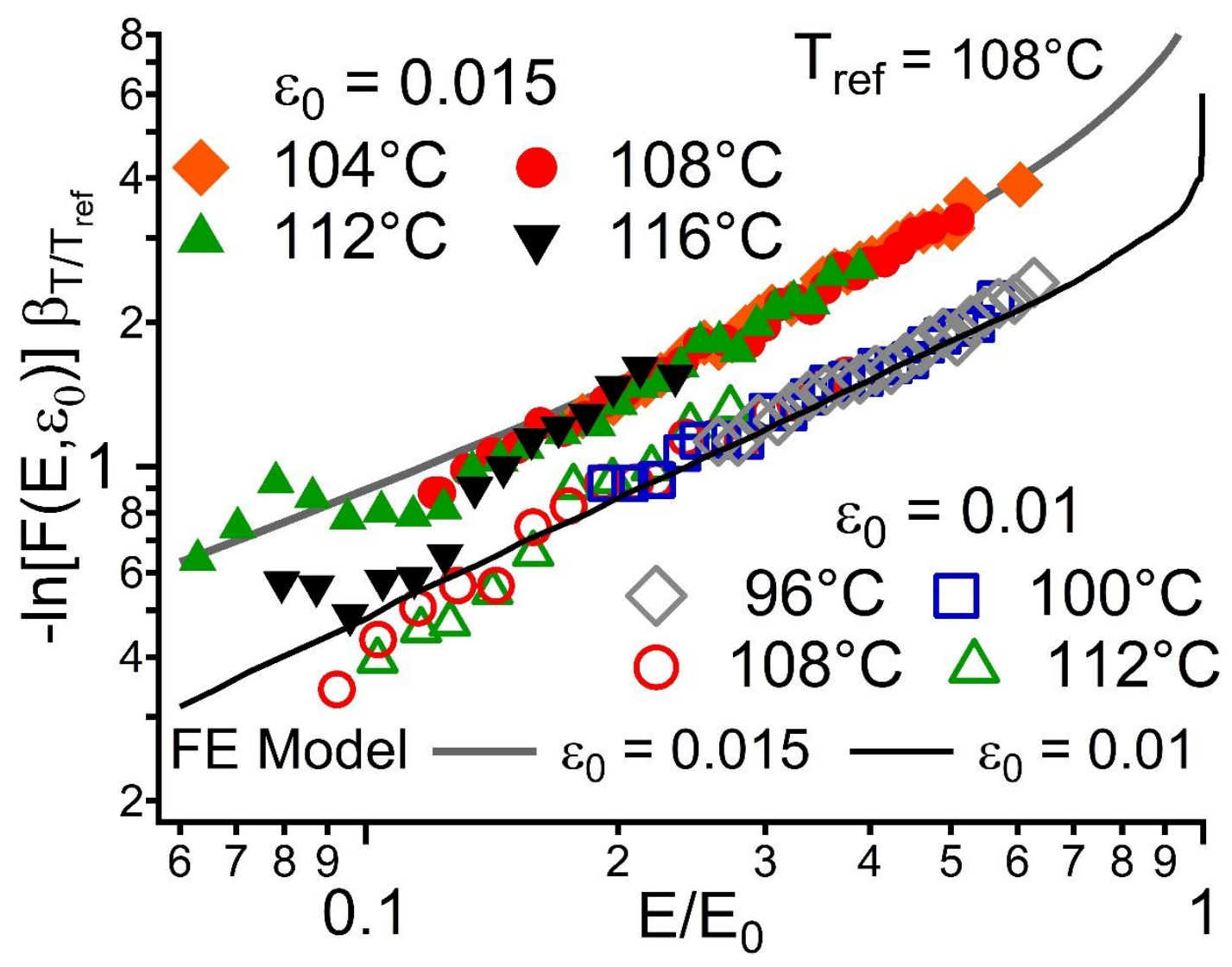

Figure 10: The quantity $-\ln \left[F\left(E, \varepsilon_{0}\right)\right]$ deduced from experiments vs. macroscopic stress $E \varepsilon_{0}$ in $\log -\log$ scale. The master curves are obtained at $T_{\text {ref }}=108{ }^{\circ} \mathrm{C}$ for strains of $1 \%$ and $1.5 \%$ applying a multiplicative shift factor $\beta_{T / T r e f}$ to $-\ln \left[F\left(E, \varepsilon_{0}\right)\right]$. Continuous lines are simulation results computed using $Y=5.75 \mathrm{MPa}$ and $f(\sigma)=\exp \left[-(\sigma / Y)^{2}\right]$ with $K=2 \mathrm{GPA}, E_{R}=1.5$ $M P a, E_{G}=1.200 G P a, s=4.83$, and $\ln \left(\tau_{0}\right)=4.1$.

In the frame of our model, the vertical shift observed between the F functions measured at different temperatures can be attributed to the temperature dependence of the critical stress $Y$. According to Long et al. [28], the parameter Y of the local acceleration function depends on the temperature and size of dynamical heterogeneities. According to the relation proposed by Long et al., we estimate the size of dynamical heterogeneities.

\subsection{Size of dynamical heterogeneities}

In the previous sections, we identified the values of $\mathrm{Y}$ to fit with the model nonlinear responses measured at different temperatures for a given deformation amplitude. Applying the relation suggested by Long et al. [28] that gives 
$Y^{2}=\frac{2 k_{B} T G_{G}}{\xi^{3}}$ and considering $\mathrm{G}_{\mathrm{G}}=427 \mathrm{MPa}$ and $\mathrm{K}=2 \mathrm{GPa}$, we found that the size of heterogeneity $\xi(\mathrm{T})$ decreases from $6.5 \mathrm{~nm}$ to $4.5 \mathrm{~nm}$ for a temperature increase of approximately $15 \mathrm{~K}$ in the temperature range probed by our experiments, as shown in the inset of figure 3 .

\section{Conclusion}

In conclusion, by combining experiments and a numerical approach accounting for dynamical heterogeneities, we show that experimental relaxation measurements preformed in the weak nonlinear regime before yielding can be described in our model by applying the same local acceleration law for all the dynamical heterogeneities. The microscopic time distribution under strain are in agreement with experimental observations of Lee et al [19]. The macroscopic responses predicted by our model using the expression of Long et al. [28] for the local acceleration function are in good agreement with the mechanical data. These results lead us to estimate the characteristic length scale of dynamical heterogeneities, which decreases with temperature and is close to $5 \mathrm{~nm}$. Finally, we show that - under nonlinear solicitation stress-induced accelerations of the mechanical relaxation are strikingly different at the local and macroscopic scales.

\section{Annex 1:}

In 3D, the differential equation of a Zener element for a linear case is

$$
\tau_{0} \dot{\overline{\bar{\sigma}^{z}}} D+\overline{\overline{\sigma^{z}}} D=\tau_{0}\left(\mathbb{C}_{R}+\mathbb{C}_{G}\right): \dot{\overline{\epsilon^{z}}} D+\mathbb{C}_{R}: \overline{\overline{\varepsilon^{z}}} D
$$

where $\overline{\overline{\sigma^{z}}} D$ and $\overline{\overline{\varepsilon^{z}}} D$ are the time dependent deviatoric stress and strain tensors, respectively, $\mathbb{C}_{R}$ and $\mathbb{C}_{G}$ are the fourth-order glassy and rubber isotropic elasticity tensors, respectively, and $\tau_{0}$ is the relaxation time.

In the case of a relaxation test, this leads to the following equation: 


$$
\frac{d \mathbb{C}}{d t}=-\frac{1}{\tau_{0}}\left(\mathbb{C}(t)-\mathbb{C}_{R}\right)
$$

where $\mathbb{C}(t)$ is the fourth-order isotropic modulus tensor such that $\overline{\overline{\sigma^{z}}} D=\mathbb{C}(t): \overline{\overline{\varepsilon^{z}}} D$

In the nonlinear case, this is

$$
\frac{d \mathbb{C}_{N L}}{d t}=-\frac{1}{\tau_{0} f\left(\sigma^{z e q}\right)}\left(\mathbb{C}_{N L}(t)-\mathbb{C}_{R}\right)
$$

where $\mathbb{C}_{N L}$ is fourth-order isotropic nonlinear modulus tensor and $f\left(\sigma^{z e q}\right)$ is an acceleration function that depends on an equivalent stress.

Regarding the term corresponding to uniaxial elongation direction, this leads to the $1 \mathrm{D}$ equations:

$\frac{d E_{L}^{Z}}{d t}=-\frac{E_{L}^{Z}(t)-E_{R}^{Z}}{\tau_{0}}$ for linear condition and $\frac{d E_{N L}^{Z}}{d t}=-\frac{E_{N L}^{Z}(t)-E_{R}^{Z}}{\tau_{0} f\left(\sigma^{z e q}\right)}$ for nonlinear condition, where $E_{L}^{Z}$ and $E_{N L}^{Z}$ are the Young's moduli in the traction direction and $E_{R}^{Z}$ is the rubber modulus.

\section{Annex 2}

Using a general local acceleration function $f(\sigma)=\exp \left[-(\sigma / Y)^{n}\right]$ with values of $\mathrm{n}$ different from 2 , the curves $-\ln \left[F\left(E, \varepsilon_{0}\right)\right]$ as a function of $E$ with $\log$ scales, exhibit a scaling regime in a limited range of relaxation modulus, i.e., $-\ln (F) \propto E^{m}$. The exponent $m$ depends on the power $n$ of the local acceleration function. It further depends on the width $s$ of the relaxation time distribution. In the limit case $s=0$ which corresponds to a homogeneous case, $f=F$ and thus, $m=n$. The variations of $m$ with $\mathrm{n}$ in the local acceleration function are plotted in figure 11 for various widths of the relaxation time distribution s. For the width of relaxation time distribution $\mathrm{s}>2.3$, the slope $\mathrm{m}$ does not significantly depend on $s$. 

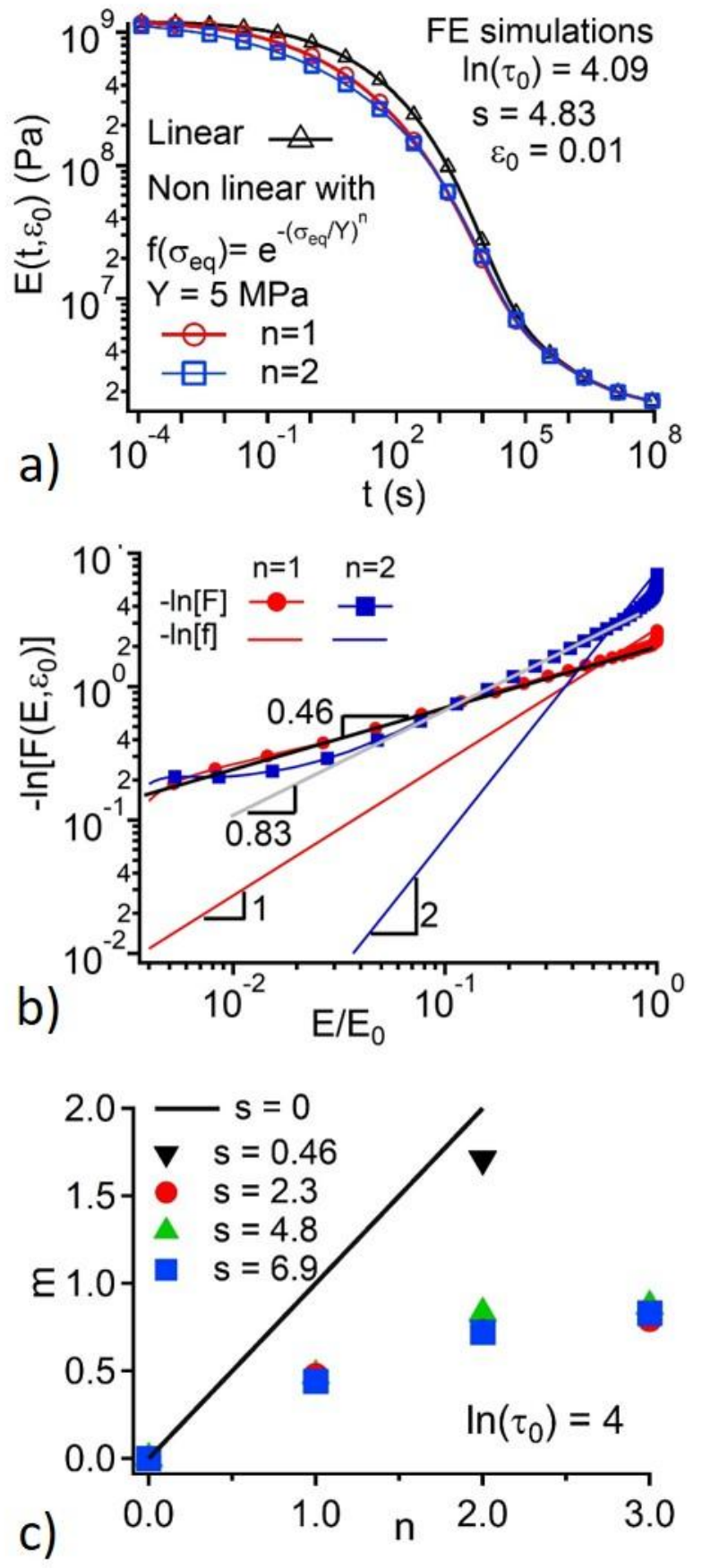

Figure 11: (a) The modulus relaxation $E\left(t, \varepsilon_{0}\right)$ as a function of time computed assuming $s=$ 4.83, $\ln \left(\tau_{0}\right)=4.1, E_{G}=1.2 \mathrm{GPa}, E_{R}=1.5 \mathrm{MPa}$, and a local stress dependence of the local acceleration function that follows $f(\sigma)=1$ and $f\left(\sigma_{e q}\right)=\exp \left[-\left(\sigma_{e q} / Y\right)^{n}\right]$ with $Y=5 \mathrm{MPa}$ for linear and nonlinear conditions, respectively. Computation were performed for $n=2$ and $n=$ 1. (b) The quantity $-\ln \left[F\left(E, \varepsilon_{0}\right]\right.$ as a function of the modulus normalised by $E_{0}$ in $\log -\log$ scale. The function F computed assuming a local stress field disorder is compared to the local acceleration function ffor different values of $n$ and $\varepsilon_{0}=0.01$. (c) The variation of the 
macroscopic exponent $m$ as a function of the local exponent $n$ for different time distribution widths $s$

\section{Reference}

[1] J. F. Peters, M. Muthuswamy, J. Wibowo, and A. Tordesillas, Phys. Rev. E 72, 041307 (2005)

[2] G. Gioia, Y. Wang and A.M. Cuitigno Proc. R. Soc. Lond. A 457, 1079 (2001)

[3] D. R. Reid, N .I. Pashine, J. M. Wozniak, H. M. Jaeger, A. J. Liu, S. R. Nagel, and J. J. de Pablo, PNAS 115, E1384 (2018)

[4] A. Dequidt, ,L. Conca, J. Y. Delannoy, , P. Sotta, F. Lequeux, F and D. R. Long, Macromolecules 49, 9148 (2016)

[5] J.L. Barrat and J. J. De Pablo, MRS BULLETIN 32, 941 (2007)

[6] C. Fusco, D. R Long and Loïc Vanel, J Stat. Mech. 053301 (2019)

[7] V. Negi and R.C. Picu, Mechanics of Materials 120, 26 (2018)

[8] F. Fujara, B. Geil, H. Sillescu, and G. Fleischer, Z. Phys. B 88, 195 (1992).

[9] M.D. Ediger, Annu. Rev. Phys. Chem. 51, 99 (2000).

[10] U. Tracht, M. Wilhelm, A. Heuer and H.W. Spiess, J. Magn. Reson. 140, 460 (1999).

[11] C. E. Maloney and A. Lemaitre, Phys. Rev. E 74, 016118 (2006) 
[12] R. Masurel, S. Cantournet, A. Dequidt, D. Long, H. Montes and F. Lequeux, Macromolecules 48, 6690 (2015)

[13] I. Kriuchevskyi, J. P. Wittmer, H. Meyer and J. Baschnagel, Phys. Rev. Lett. 119, 147802 (2017).

[14] B. Shang, J. Rottler, P. Guan and J.L. Barrat, Phys. Rev. Lett. 122, 105501 (2019)

[15] L. Klochko, J. Baschnagel, J. P. Wittmer and A. N. Semenov, J. Chem. Phys. 151, 054504 (2019).

[16] L.S. Loo, R.E. Cohen and K.K. Gleason, Science 288, 116 (2000).

[17] R. Perez-Aparicio, D. Cottinet, C. Crauste-Thibierge, L. Vanel, P. Sotta, J.Y.

Delannoy, D.R. Long and S. Ciliberto, Macromolecules 49, 3889 (2016).

[18] J. Kalfus, A. Detwiler and A.J. Lesser, Macromolecules 45, 4839 (2012).

[19] H.N. Lee, R.A. Riggleman, J.J. de Pablo and M.D. Ediger, Macromolecules 42, 4328 (2009).

[20] G. Tieghi, M. Levi, A. Fellini and F. Danusso, Polymer 32, 39 (1991)

[21] P.A. O'Connell and G. B. McKenna, Polymer Engineering \& Science 37, 1485 (1997)

[22] P.A. O'Connell and G. B. McKenna, Mechanics of Time Dependent Materials 6, 207 (2002)

[23] M.C. Boyce, E.L. Montagut and A.S. Argon, Polym. Eng. Sci. 32, 1073 (1992).

[24] A. S. Argon, Phil. Mag. 28, 39. (1973) 
[25] H. Eyring, J. Chem. Phys. 4, 283 (1936).

[26] S. Langer, Phys. Rev. E 77, 021502 (2008).

[27] H.-N. Lee, K. Paeng, M. D. Ediger, R. Stamm, G. Medvedev and J. Caruthers, J. of Polym. Sci. Part B 47, 1713 (2009)

[28] D. Long, L. Conca, L. and P. Sotta, Phy. Rev. Mat. 2, 105601 (2018)

[29] F. Casas, C. Alba-Simionesco, F. Lequeux, H. Montes, J. Non Cryst. Solids, 352, 5076 (2006)

[30] A. Belguise, S. Cantournet, F. Lequeux and H. Montes in Mechanics and Physics of Solids at Micro- and Nano-Scales edited by I. R. Ionescu, S. Queyreau, C. R. Picu, O. U. Salman (ISTE Ldt, London and John Wiley \& Sons, USA, 2019) p. 231

[31] R. J. Masurel, P. Gelineau, , S. Cantournet, S., A. Dequidt,,D. R. Long, F. Lequeux, , H. Montes. Phys. Rev. Lett. 118, 047801 (2017)

[32] R. J. Masurel, P. Gelineau, F. Lequeux, S. Cantournet, H. Montes, Eur. Phys. J. E (2017) 40,116

[33] J. Rottler, M.O. Robbins, Phys. Rev. Lett. (2005) 95, 225504.

[34] I.M. Ward, Mechanical Properties of Solid Polymers (John Wiley, 1983).

[35] Z-set: http://www.zset-software.com/.

[36] D. Ryckelynck, F. Vincent, S. Cantournet, Comput. Methods Appl. Mech. Eng. 225, 28 (2012). 
[37] Livermore C and Voldman J 2004 Material Properties Database http://www.mit.edu/ 6.777/matprops/matprops.htm

[38] R. Colby M. Rubinstein, Polymer Physics, (Oxford University Press 2003) 